\title{
Abdominal and pelvic imaging in the diagnosis of acute abdominal attacks in patients with hereditary angioedema due to C1-inhibitor deficiency
}

\author{
Piotr Obtułowicz ${ }^{1}$, Marcin Stobiecki²,3, Wojciech Dyga ${ }^{2,3}$, Aldona Juchacz ${ }^{4}$, Tadeusz Popiela ${ }^{1,5}$, Krystyna Obtułowicz ${ }^{2,3}$ \\ 'Department of Diagnostic Imaging (NSSU), University Hospital, Krakow, Poland \\ 2Department of Clinical and Environmental Allergology, Jagiellonian University Medical College, Krakow, Poland \\ ${ }^{3}$ Hereditary Angioedema Centre, University Hospital, Krakow, Poland \\ ${ }^{4}$ The Greater Poland Centre for Pulmonology and Thoracic Surgery, Poznan, Poland \\ ${ }^{5}$ Department of Radiology, Jagiellonian University Medical College, Krakow, Poland
}

\begin{abstract}
Introduction: Hereditary angioedema (HAE) is a rare inherited autosomal dominant disease caused by deficiency or dysfunction of $\mathrm{C} 1$ inhibitor (C1INH). Clinical symptoms include recurrent subcutaneous and submucosal angioedema of the internal organs. Abdominal attacks affect more than $90 \%$ of patients, are often misdiagnosed and result in unnecessary surgical procedures.

Aim: To analyse the utility of imaging studies (USG, CT) in patients with C1INH-HAE during an abdominal attack and remission.

Material and methods: We enrolled 40 patients with type I and II HAE (30 women, 10 men; mean age 39 years). The diagnosis of C1INH-HAE was based on patient and family history, significantly reduced values of C1INH serum level and activity. Abdominal and pelvic ultrasound were performed in patients within the first $6 \mathrm{~h}$ of the abdominal attack and repeated during remission. Moreover, 23 cases underwent abdominal or pelvic computed tomography during acute abdominal symptoms. The most common ultrasound and CT findings showed the transient presence of a significant amount of fluid in the free abdominal cavity and intestinal oedema during the symptom progression and spontaneously disappearing during the seizure in $90 \%$ and $50 \%$ of patients, respectively. CT revealed also an enlargement of the mesenteric lymph nodes as well as a fat stranding along the bowel wall thickening. Conclusions: Ultrasound or CT imaging facilitates the diagnosis of the patient suspected of having an abdominal attack due to C1INH-HAE. They allow to identify transitional presence of an abundant fluid in the free abdominal cavity and intestinal swelling which spontaneously disappear with a symptoms attack.
\end{abstract}

Key words: abdominal imaging, bradykinin, $\mathrm{C} 1$ inhibitor, hereditary angioedema.

\section{Introduction}

Hereditary angioedema (HAE) is a rare inherited autosomal dominant disease caused by deficiency or dysfunction of $\mathrm{C} 1$ inhibitor (C1INH) [1-6]. There are two phenotypes of the disease. Type I affects about $85 \%$ of cases and is characterized by significantly reduced levels and functional activity of C1INH (by more than 50\% compared with normal values). On the other hand, in type II, serum C1INH levels are normal or slightly elevated, and only the activity is largely reduced. The clinical course and symptoms of the disease are similar in both types. Symptoms include recurrent self-limited subcutaneous oedema (typically of the hands, feet, face, and genitals); submucosal oedema affecting the internal organs such as the upper respiratory tract (throat, larynx), which may be life-threatening; or gastrointestinal oedema manifesting as recurrent abdominal pain, often mimicking acute abdomen. Oedema is caused by hereditary $\mathrm{C} 1 \mathrm{INH}$ deficiency due to Serping1 gene mutation, which predisposes these patients to bradykinin overproduction, which

Address for correspondence: Wojciech Dyga, Hereditary Angioedema Centre, University Hospital, Krakow, Poland,

e-mail: wojciech.dyga@uj.edu.pl

Received: 27.05.2021, accepted: 10.06.2021. 
induces swelling reactions due to various stimuli [7-9]. Abdominal symptoms constitute a significant diagnostic challenge, with the necessity to exclude numerous other causes of acute abdominal pain. Angioedema attacks at other sites are rare and may affect the urinary tract (bladder, urethra, kidneys), musculoskeletal system as well as pericardial or pleural cavity. Attacks may also present with neurological manifestations such as headaches, transient vision loss, and migraine-like symptoms $[5,6]$.

Abdominal attacks constitute an important problem in HAE because they affect more than $90 \%$ of patients and occur even up to 2 to 3 times a month. They may be the first manifestation of angioedema in patients with $\mathrm{C} 1 \mathrm{INH}-\mathrm{HAE}$ and may remain the only symptom of the disease for many years [5, 10-13]. They often lead to significant delays in accurate diagnosis.

Acute abdominal attacks are common in the general adult population as well as in paediatric patients [5, 14]. Each time, it is necessary to exclude other possible causes, such as appendicitis, diverticulitis, sigmoiditis, mesenteric lymphadenitis, intussusception, bowel obstruction, polycystic ovary syndrome, ovarian or testicular torsion, and intestinal bleeding or ischemia $[3,5,10]$. The diagnosis of an abdominal attack in HAE is based on medical history, including C1INH deficiency confirmed by a laboratory test (significant reduction in C1INH serum level and C1INH activity in plasma compared with normal) $[4,15]$. The deficiency may be accompanied by reduced C4 level, especially during the attack. Patients may report abdominal attacks lasting several days and resolving spontaneously after 3 to 5 days or after $\mathrm{C} 1 \mathrm{NNH}$ administration. A positive family history is also commonly reported [3, 5, 6].

The diagnosis of patients with C1INH-HAE and an abdominal attack is a considerable challenge at emergency,

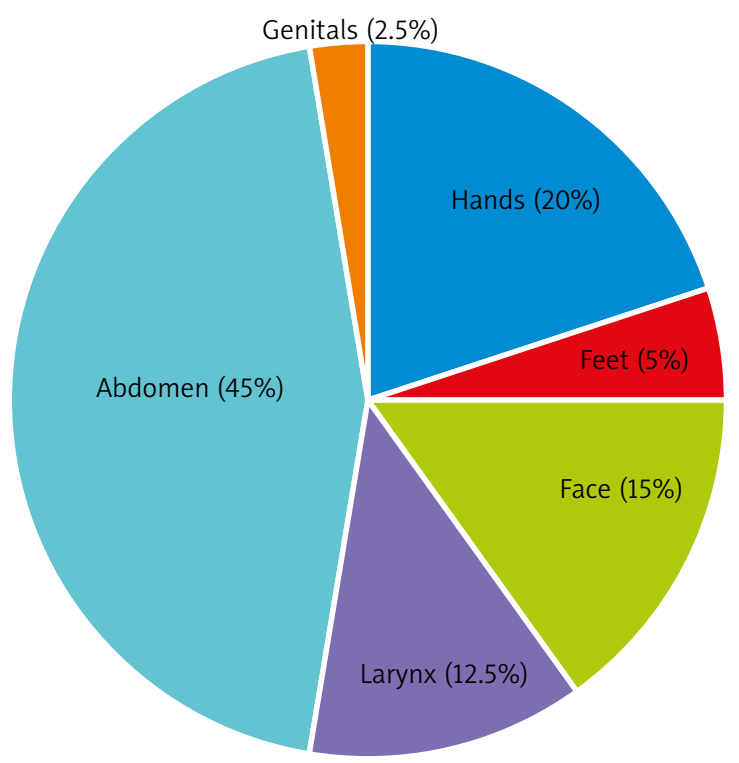

Figure 1. Parts of the body affected by edema during the first hereditary angioedema attack. Data presented as percentage of cases $(n=40)$ surgical, and gastroenterological units, which often results in unnecessary surgical procedures such as exploratory laparotomy [3, 5, 10, 12, 16-20]. Acute abdominal attacks in the course of HAE often manifest with acute abdominal pain with cramps, progressing over time and reaching the highest severity after several hours from onset without concomitant peritoneal symptoms. Pain is often accompanied by nausea, vomiting, severe flatulence, or less frequently, diarrhoea. Patients may also present with significant weakness, most often caused by the presence of excess peritoneal fluid due to hypovolemic shock [5, 21].

In patients with confirmed C1INH deficiency, the diagnosis of an abdominal attack may be aided by the administration ex juvantibus of plasma-derived $\mathrm{C} 1 \mathrm{INH}$, recombinant human $\mathrm{C} 1 \mathrm{INH}$, bradykinin $\mathrm{B} 2$ receptor antagonist - icatibant, or plasma kallikrein inhibitor ecallantide or also fresh frozen plasma. The effectiveness of these drugs within $1 \mathrm{~h}$ from administration confirms an abdominal attack in C1INH-HAE [3, 5, 22].

\section{Aim}

The aim of this retrospective study was to assess the diagnostic utility of abdominal and pelvic imaging (ultrasound, computed tomography (CT)) in patients with $\mathrm{C} 1 \mathrm{INH}-\mathrm{HAE}$ during an abdominal attack and remission. We also aimed to assess data from medical history and physical examination performed during an attack to identify any potential criteria that could aid the diagnosis of HAE attack, facilitate the interpretation of imaging studies, and help assess patient eligibility for ultrasound or CT imaging during an attack.

\section{Material and methods}

The study included 40 patients with C1INH-HAE and abdominal attacks (30 women and 10 men; mean age: 39 years; range: $19-70$ years). The diagnosis of $\mathrm{C} 1 \mathrm{NNH}-$ HAE was based on the presence of previous subcutaneous or submucosal angioedema attacks, a positive family history, and the measurement of plasma C1INH activity, serum $\mathrm{C} 1 \mathrm{INH}$ and $\mathrm{C} 4$ concentration.

Type $1 \mathrm{CIINH}-\mathrm{HAE}$ was diagnosed in 34 patients on the basis of a reduction of serum $\mathrm{C} 1 \mathrm{INH}$ concentration and plasma C1INH activity below $50 \%$ as compared to normal values. Type II C1INH HAE was diagnosed in 6 patients with normal value of serum C1 INH concentration and plasma C1INH activity below $50 \%$ as compared to the normal limit - according to standard criteria [2, 5, 15]. Diagnostic laboratory studies were performed during remission using the nephelometric method with a BN100 Nephelometer (Dade Behring). Functional $\mathrm{C} 1 \mathrm{NH}$ activity was measured in plasma with the colorimetric kinetic method, using a chromogenic assay (Berichrom C1Inhibitor and Complement Reagents) and Behring Coagulation Timer analyzer (both Dade Behring).

Of the study group, 30 (75\%) patients had a positive family history. The first angioedema attack occurred be- 

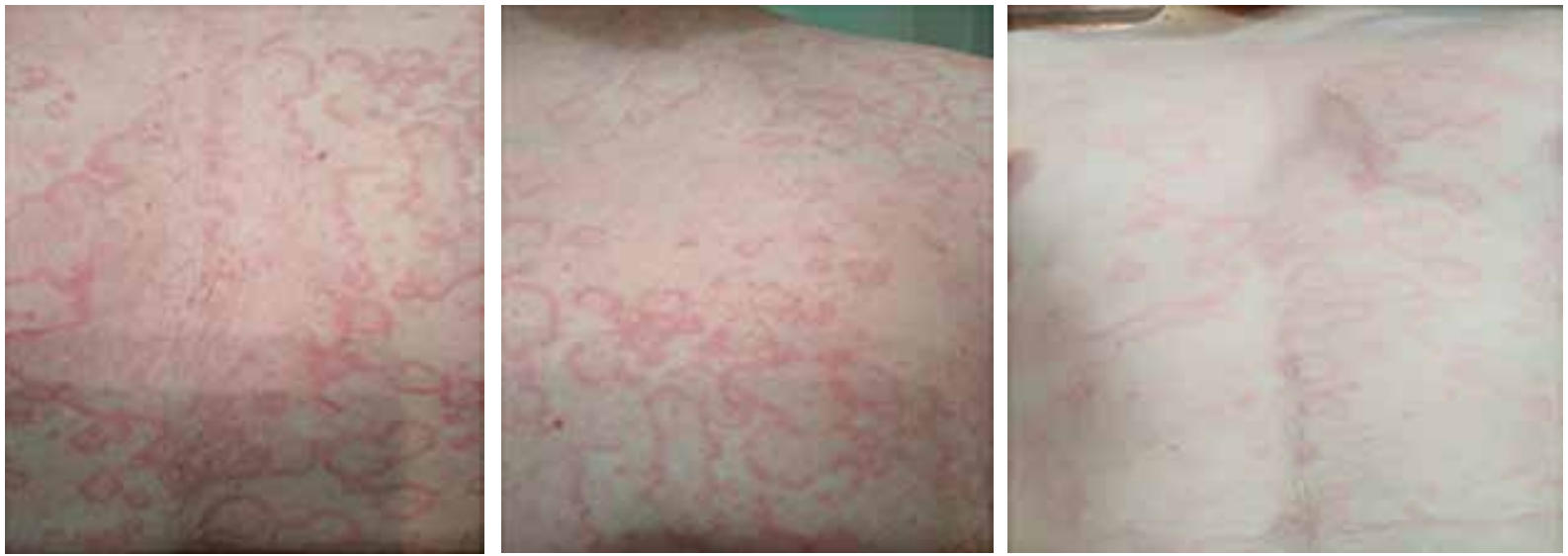

Figure 2. Erythema marginatum

tween the age of 2 and 29 years. The diagnosis of HAE was established 1 to 41 years after the first attack. Organ involvement at the first episode (Figure 1) was as follows: abdomen in 18 (45\%) patients, hands and feet in 10 (25\%), face in 6 (15\%), larynx in 5 (12.5\%), and genitals in 1 (2.5\%) patient. Moreover, 19 patients (47.5\%) developed erythema marginatum (Figure 2) before the attack and independently of the site of skin involvement (most often on the chest). However, it resolved spontaneously during the attack.

We retrospectively assessed 84 ultrasounds of the abdominal and lower pelvic regions. Ultrasound examination was performed in patients during an abdominal attack and remission. In 10 patients, imaging studies were performed more than once (2-9 times) during different attacks. Moreover, we assessed abdominal and pelvic CT scans obtained simultaneously with ultrasound in 23 patients during acute abdominal attacks. This retrospective analysis covers imaging tests that were performed during approximately 15 years at different diagnostic imaging units by different clinicians.

\section{Results}

The results of this retrospective analysis of imaging tests performed during acute abdominal attacks are presented in Figures 3, 4 A, B, 5 A, B and 6.

Abdominal and pelvic ultrasound performed in the first hours of acute abdominal attacks (Figure $4 \mathrm{~A}$ ) in 36 (90\%) patients revealed free peritoneal fluid. In some patients, low fluid volume was present in single peritoneal pouches in the pelvis, while in others, diffuse fluid accumulation was observed in the peritoneal cavity and additionally in several pouches, most commonly the hepatorenal recess, subhepatic, and pelvic peritoneal space. Segmental bowel wall thickening was much less common (identified in about $30 \%$ of patients). In 2 cases, the only abnormality on ultrasound was mesenteric lymph nodes, although without enlargement. In all patients, an ultrasound performed at day 3 of symptom remission revealed resolution of all abnormalities observed during the attack. A comparison of ultrasounds obtained several times during different attacks showed differences in findings in terms of the site and extent of intestinal oedema or fluid volume. Medical history confirmed that the disease course and severity of symptoms also differed between these individual episodes.

Abdominal and pelvic CT performed during an attack (Figure 4 B) showed similar abnormalities to those revealed by ultrasound, but it enabled a more accurate assessment. As with ultrasound, the most common finding was free peritoneal fluid ( $80 \%$ of patients). However, the presence of intestinal oedema was more frequent and more easily identified by CT scans. It also outperformed

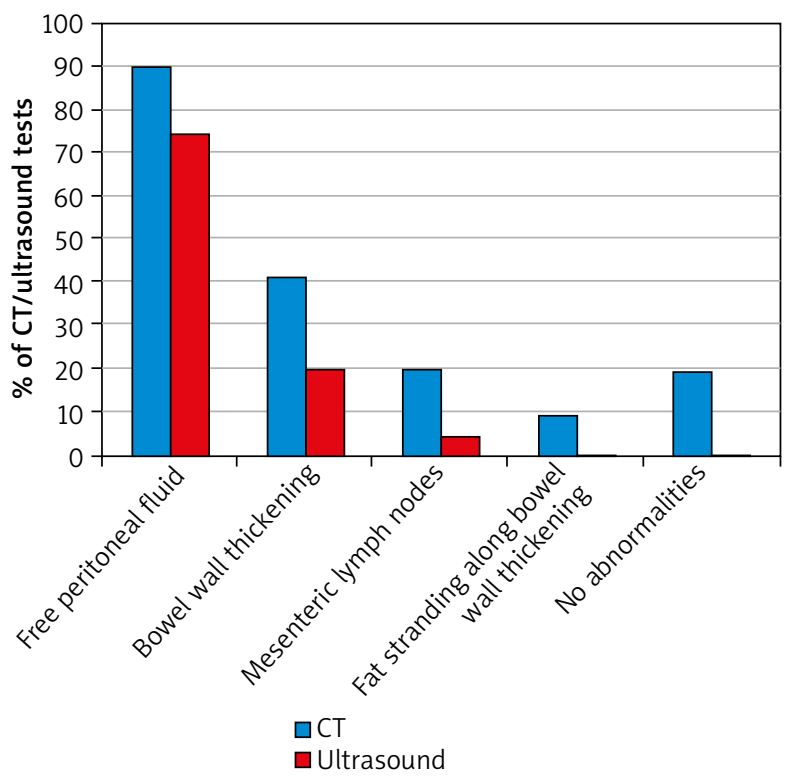

Figure 3. Abdominal and pelvic ultrasound and computed tomography finding during acute abdominal attack in the course of hereditary angioedema 

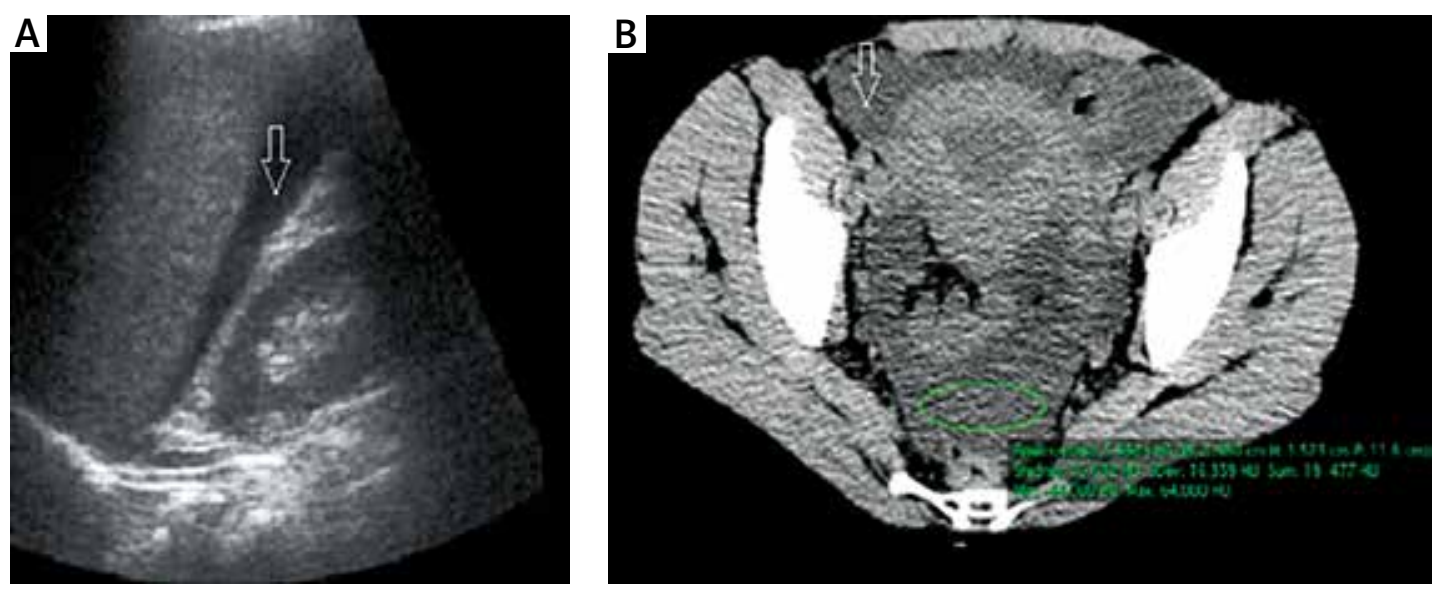

Figure 4. Free peritoneal fluid (arrow) on ultrasound (A) and computed tomography (B)
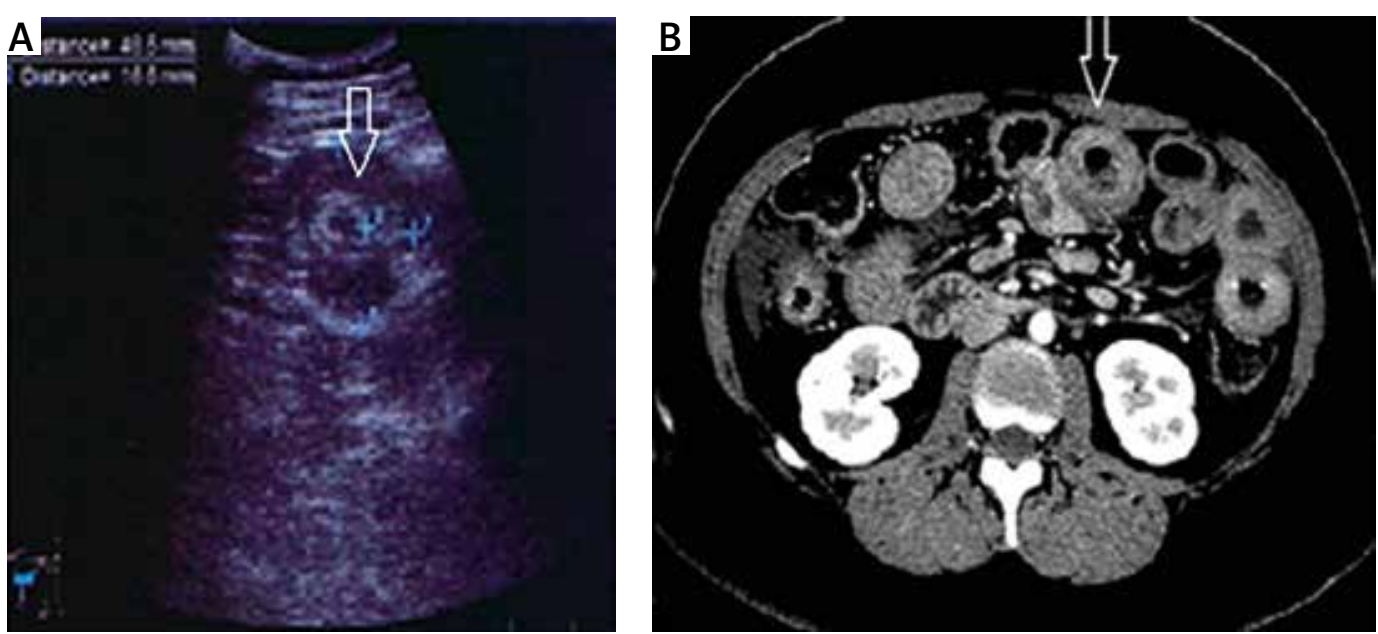

Figure 5. Bowel wall thickening (arrow) on ultrasound (A) and computed tomography (B)

ultrasound in terms of assessing the morphology of the intestinal wall and mucosal swelling (such as assessment of circular wall thickening, bowel wall stiffness, or multifocal oedema (e.g., affecting several bowel loops)). Finally, CT also enabled a more detailed evaluation of lymph nodes and fat stranding along bowel wall thickening (which is not visible on ultrasound).

Peritoneal fluid on ultrasound is typically homogeneous and lacks echogenicity, while CT reveals low density (approximately 5-20 jH). A laboratory analysis of intraoperative fluid performed in 5 patients revealed the features of a transudate, water-like, and sterile fluid with low neutrophil and mononuclear counts.

Bowel wall thickening involved various segments, most commonly, the loops of the jejunum, followed by those of the ileum, duodenum, and colon. Both ultrasound and CT revealed 2 distinct types of swelling (Figures $5 \mathrm{~A}, \mathrm{~B}$ and 6):

1) symmetrical segmental thickening of all small bowel wall layers, with high attenuation of the inner and outer layers representing the mucosa and muscularis propria, respectively, corresponding to the so-called Target sign. This radiologic appearance of the bowel wall thickening should be differentiated from intestinal lymphoma and inflammatory bowel disease, among other conditions.

2) Segmental thickening of the small bowel mucosal folds due to submucosal oedema of the folds that run perpendicular to the long axis of the bowel (so-called stack of coins sign) and due to intramural blood accumulation secondary to submucosal haemorrhage and oedema (so-called thumbprint sign), or diffuse bowel wall thickening. The differential diagnosis should include intestinal ischemia, Henoch-Schönlein purpura, or intramural haemorrhage (due to trauma, haemophilia, or anticoagulant therapy).

Cases with notable bowel wall thickening on CT also showed concomitant fat stranding suggesting oedema and hyperaemia of the adjacent fat tissue and vessels. These oedematous changes are segmental (self-limited) 


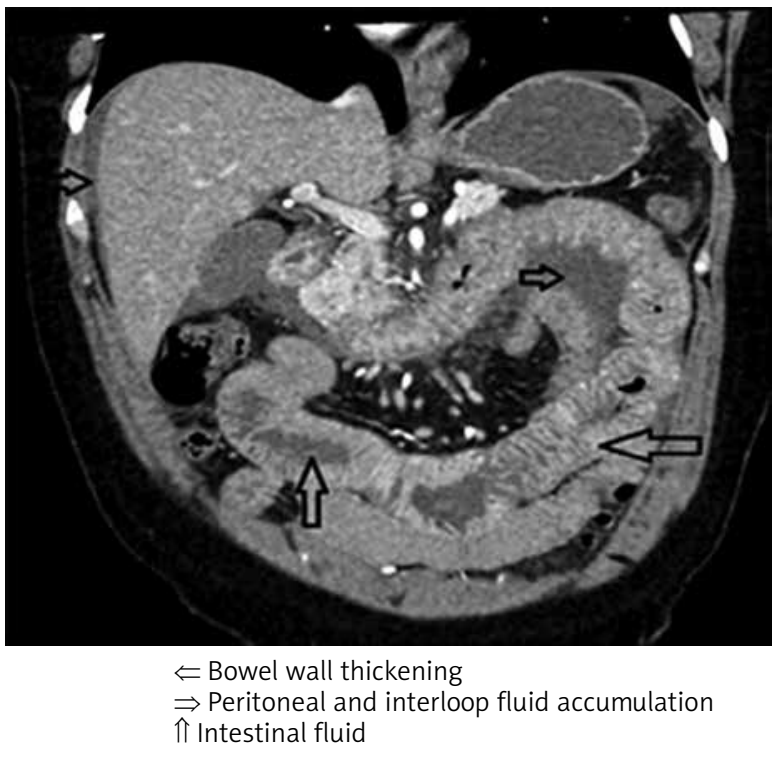

Figure 6. Computed tomography scan in a patient with abdominal attacks with C1INH-HAE

and can be observed only during acute symptoms as motility disorders without clinical features of bowel obstruction.

Another ultrasound and CT sign of an abdominal attack in our patients was nonspecific swelling of the mesenteric lymph nodes, although without enlargement, which resolved spontaneously after the attack. It was observed on ultrasound in 10\% of patients, and on CT, in 30\% (Figure 3).

A detailed medical history allowed us to establish several criteria suggesting an abdominal attack in a patient with HAE. In 12 (30\%) patients, an abdominal attack was accompanied by external swelling. Some of the patients reported the attack to be induced by the following factors: an abundant meal, starvation, dietetic error, excessive physical exercise, and stress. In some patients, the attack was preceded by prodromal symptoms such as bad mood, sleepiness, and irritability. Moreover, 19 (47.5\%) patients developed erythema marginatum several hours before the attack. It slowly progressed over time and resolved during the attack. Medical history revealed that in our patients the most acute abdominal attack usually manifested with recurrent acute pain, which worsened over time, reached maximum severity after several hours, and persisted for about $24 \mathrm{~h}$, then spontaneously resolved gradually over 2 to 3 days or $1-2 \mathrm{~h}$ after treatment (Figure 7).

Recurrent pain was associated usually with nausea/vomiting in 34 (85\%) patients, watery diarrhoea in $18(45 \%)$ patients, and severe bloating in 30 (75\%) patients. In addition, 6 (15\%) patients reported considerable weakness with hypotension during acute attacks with vomiting, diarrhoea, and significant ascites. Symptoms

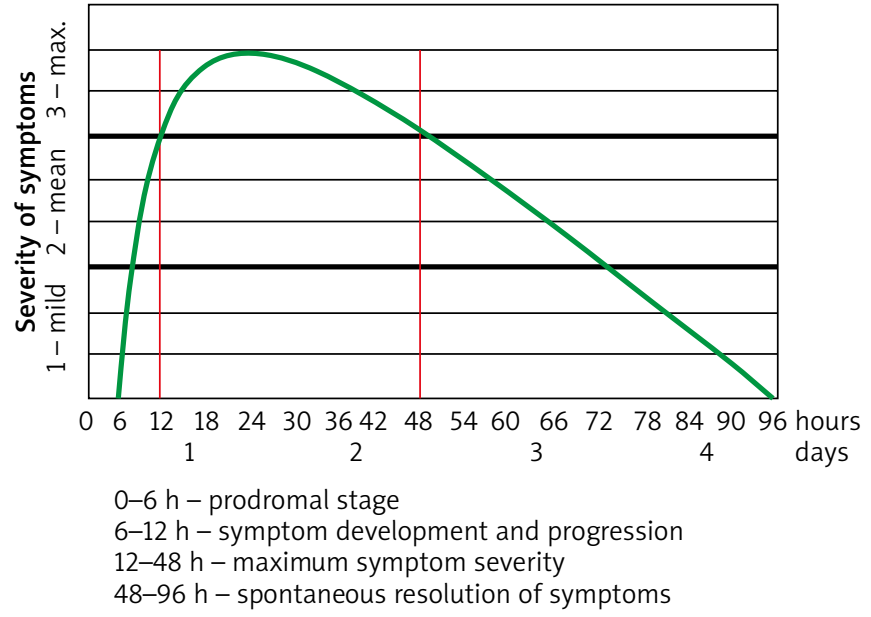

Figure 7. Stages and course of abdominal attacks in patients with hereditary due to $\mathrm{C} 1$ inhibitor deficiency

persisted for $24 \mathrm{~h}$. Finally, in some cases the administration ex juvantibus of plasma derived C1INH or fresh frozen plasma was reported to stop symptom progression within $1 \mathrm{~h}$ and to result in complete symptom resolution within a few hours in patients with C1INH-HAE.

The analysis of medical history, physical examination performed during progression of abdominal symptoms, as well as medical records revealed the following findings:

1) Several recurrent abdominal attacks in the past, often with recurrent ascites. They resolved spontaneously without treatment, usually gradually after 2 to 3 days from onset or gradually within several hours from $\mathrm{C} 1 \mathrm{INH}$ or fresh plasma administration.

2) In 14 of the 40 (35\%) patients, exploratory laparotomy was performed during the attack. In 8 cases, it revealed excess peritoneal fluid but did not identify the cause of the attack. In 5 cases, laparotomy revealed a concomitant cause of acute abdomen.

3) The analysis of additional laboratory tests performed in patients during an acute abdominal attack frequently revealed an increased white blood cell count and haematocrit, reduced activated partial thromboplastin time, and less frequently, highly elevated D-dimer levels (without radiologic signs of vascular coagulation). The least common finding was an increased C-reactive protein level.

As mentioned above, exploratory/diagnostic laparotomy was performed in 13 of the 40 patients (33\%). In 8 cases, no surgical cause of acute abdominal pain was found. It only revealed the presence of free peritoneal fluid and, rarely, segmental bowel thickening. However, in 5 cases, concomitant causes of acute abdomen were found, including acute appendicitis in 3 patients, ruptured ovarian cyst in 1 patient, and duodenal ulcer perforation in 1 patient (perforation causing acute abdomen 
occurred on the third day of recurrent abdominal attacks in the course of $(1 \mathrm{INH}-\mathrm{HAE}$ ).

\section{Discussion}

Acute abdominal attacks and external swelling are the most common symptoms of C1INH-HAE.

According to the results of our research, attacks occur in more than $90 \%$ of patients with $\mathrm{C} 1 \mathrm{INH}-\mathrm{HAE}$ and constitute a considerable diagnostic challenge for emergency clinicians, surgeons, gastroenterologists, and gynaecologists, requiring a differential diagnosis with numerous other possible conditions presenting with acute abdomen [5, 10, 14, 17, 19, 20, 23-26].

Therefore, patients are commonly misdiagnosed and receive inappropriate treatment including unnecessary exploratory laparotomy [3, 5, 10, 12, 16-20, 23]. Finally, abdominal attacks cause long delays in diagnosis, even up to several years, especially when they are the only presenting symptom of $\operatorname{HAE}[11,13,19,20,27-31]$. Therefore, numerous investigators have emphasized the clinical value of abdominal and pelvic imaging and the necessity to introduce these modalities into regular diagnostic workup of patients with abdominal attack in the course of C1INH-HAE [3, 5, 6, 23, 26, 32-34].

Our analysis of abdominal and pelvic imaging (ultrasound and $\mathrm{CT}$ ) in patients with an abdominal attack in the course of $\mathrm{C} 1 \mathrm{INH}-\mathrm{HAE}$ revealed 2 characteristic findings, namely, the presence of free peritoneal fluid (in more than $90 \%$ of patients) and segmental bowel wall or mucosal thickening (in about $50 \%$ of patients). These observations are consistent with the results of other studies to date $[5,6,10,11,17,18,27,34-38]$.

In addition, evaluation of imaging findings as well as medical history data allowed us to identify several characteristic features of free peritoneal fluid. These included variable fluid volume over subsequent attacks, which correlated with pain symptoms, as well as its spontaneous resolution or resolution after ex juvantibus treatment [3, 5, 10, 22, 23]. According to Agostoni et al. [6] and the results of our findings, the appearance of fluid on ultrasound or CT imaging during an abdominal attack in patients with C1INH-HAE depends on its volume. Small fluid accumulation is often visible in the subhepatic/subsplenic region and always in the pouch of Douglas. On the other hand, larger fluid volume can be seen in the perisplenic region and between bowel loops (which are often oedematous and thickened). Freely floating loops suggest excessive fluid volume.

The presence of fluid during an abdominal attack in patients with HAE requires exclusion of other causes, such as dissemination of neoplastic disease, decompensated cirrhosis of the liver inflammatory diseases, nephrotic syndrome, protein-losing enteropathy, or mesenteric venous thrombosis. An important aspect to consider in the differential diagnosis is the rate of fluid ac- cumulation and resolution. Significant ascites in a patient with an abdominal attack may be complicated by severe weakness. This results in hypovolemic shock if a large volume of fluid leaks into the intestines and peritoneal cavity, especially when accompanied by vomiting or diarrhoea (which can be easily established on the basis of medical history) [5, 10, 21, 22, 29, 34, 35, 37, 39]. In such cases, apart from excess free fluid in the abdomen or pelvis, imaging reveals also the leakage of watery fluid into the intestines, which results in watery diarrhoea seen in many patients during an attack.

As stated above, another characteristic radiologic finding in patients with C1INH HAE during an abdominal attack was segmental oedema of bowel wall or mucosal thickening, seen on ultrasound in $25 \%$ of patients and on CT in $50 \%$ of patients. It involved all layers of the bowel wall over a segment of more than $10 \mathrm{~cm}$ in length. Most often, it was localized in the small bowel, which is in line with the results of previous studies [5, 6, 33-35].

Exclusion of mesenteric venous thrombosis in patients with HAE and an abdominal attack is a common problem associated with the presence of elevated Ddimer levels, especially in attacks related to abnormalities of the kinin, coagulation and fibrinolysis systems in patients with bradykinin-mediated angioedema typical for patients with C1INH-HAE [10, 23, 40, 41].

About $20 \%$ of patients with acute abdominal attack did not present with typical findings on CT. This may be due to the fact that CT study was performed too early or too late, because the presence of fluid during an attack in patients with C1INH-HAE is transient and resolves spontaneously with other symptoms of the attack $[5,10]$. In 8 cases, abdominal CT revealed single nonspecific and low amounts of fluid in the bowels. In 2 patients, the radiologic diagnosis of partial intestinal obstruction was not confirmed by subsequent surgical follow-up.

Follow-up ultrasound and CT performed during spontaneous remission or after treatment revealed complete resolution of abnormalities observed during an attack, which is in line with other studies [5, 6, 23, 33]. Therefore, recurrent fluid and its spontaneous resolution as well as segmental bowel thickening are considered to be the typical symptoms of an abdominal attack in patients with HAE. Medical history and records obtained from these patients often reveal recurrent attacks with ascites, lasting a few days, and resolving spontaneously, which can be of great help in assessing ultrasound and CT images in an abdominal attack in a patient with $\mathrm{C} 1 \mathrm{INH}-\mathrm{HAE}$ as well as in qualification to perform these tests.

Considering the above, recurrent ascites during progression and the most acute stage of abdominal pain, which resolves during the attack, may be a specific sign or symptom of an abdominal attack in patients with C1INH-HAE [5, 10, 34-37]. Therefore, when evaluating abdominal ultrasound or CT findings during an abdominal attack in patients with C1INH-HAE or in patients with 
a suspicion of acquired C1INH deficiency in the course of other conditions [2-6, 10, 32-35, 37], it is important to remember that the presence and amount of free $a b$ dominal fluid is variable and transient. The fluid may be completely absent or present only in small amounts, both in the early stage of the attack and during resolution of abdominal symptoms.

As stated above, when interpreting abdominal imaging findings or referring patients for imaging tests to establish the cause of an abdominal attack in patients with C1INH-HAE, it is especially important and valuable to consider data from medical and family history, physical examination of the patient during an attack, as well as the characteristic presentation of the attack itself. The typical clinical signs and symptoms that can be recognized on the basis of medical history and that fulfil the diagnostic criteria for an abdominal attack in C1INH-HAE include recurrent abdominal attacks lasting several days, often with concomitant vomiting, and resolving gradually and spontaneously within 3 to 5 days. They may be accompanied by localized external swelling or erythema marginatum. The diagnosis is facilitated by a positive family history or confirmed C1INH deficiency in medical records. Medical history may also report the effectiveness of plasma-derived $\mathrm{C} 1 \mathrm{INH}$ administered during the attack $[5,22]$.

The analysis of data from exploratory laparotomies performed during an abdominal attack in a patient with C1INH-HAE may be particularly valuable. In our study, laparotomy was performed in $33 \%$ of patients. In 8 cases, no surgical cause of acute abdominal attack apart from ascites and, less frequently, segmental bowel wall thickening was revealed. This confirms that laparotomy is often unnecessary in these patients, as previously reported by other authors [5, 6, 10, 19, 20, 23]. Patients with C1INH-HAE with isolated abdominal attacks constitute an important diagnostic challenge [23, 27-29, 35, 37]. In our study, an abdominal attack was the first, and for some time the only, symptom of HAE in as many as 45\% of patients with C1INH-HAE, often children, which is in line with previous reports $[5,6,14]$. Our results as well as available literature data allow us to assume that an abdominal attack in C1INH-HAE may be recognized using ultrasound imaging on the basis of segmental bowel wall thickening as well as significant fluid accumulation, both resolving spontaneously, usually by the end of day 2 of the attack. Ultrasound examination is easily accessible, safe, and quick to perform. On the other hand, abdominal or pelvic CT provides more details that allow clinicians to assess the severity and extent of bowel thickening, examine mesenteric lesions, and evaluate the volume and distribution of free peritoneal fluid. Moreover, it makes it possible to exclude other potential causes of an acute abdominal attack, such as appendicitis, diverticulitis, or gastrointestinal perforation [10, 19, $20,40]$. However, CT examination is not always feasible in patients with $\mathrm{C} 1 \mathrm{INH}-\mathrm{HAE}$ with acute abdomen, who require prompt management due to a severe clinical condition, as reported previously by numerous other authors $[10,17,19,20,33,35]$.

Importantly, if C1INH-HAE is suspected in a patient with an acute abdominal attack, it is necessary to assess C1INH levels and functional activity as well as C4 level. An immediate measurement is not always possible in an acute state, but it allows the final diagnosis to be established. This is important because similar abdominal attacks may also occur (although rarely) in patients with acquired angioedema due to $\mathrm{ClINH}$ deficiency in the course of other conditions such as T-cell proliferative disease or autoimmune diseases as well as in response to angiotensin-converting enzyme inhibitors [2, 3].

\section{Conclusions}

Abdominal and pelvic ultrasound and $\mathrm{CT}$ are valuable diagnostic tools in patients with abdominal attacks in the course of $\mathrm{C} 1 \mathrm{INH}-\mathrm{HAE}$, provided that they are performed during symptom progression or highest symptom severity.

Ascites and segmental bowel thickening are the most common self-limited symptoms of abdominal attacks in patients with C1INH-HAE, and they resolve spontaneously or after administration of an appropriate treatment.

Medical history (recurrent abdominal attacks, especially with ascites, confirmed C1INH deficiency, positive family history) and the presence of external swelling as well as erythema marginatum on physical examination during an attack are useful clinical criteria for radiological recognizing an abdominal attack due to $\mathrm{C} 1 \mathrm{INH}-\mathrm{HAE}$. They may be used to aid the interpretation of imaging findings or when assessing patient eligibility for imaging tests.

\section{Conflict of interest}

The authors declare no conflict of interest.

\section{References}

1. Busse PJ, Christiansen SC. Hereditary angioedema. N Engl J Med 2020; 382: 1136-48.

2. Cicardi M, Aberer W, Banerji A, et al. Classification, diagnosis, and approach to treatment for angioedema: consensus report from the Hereditary Angioedema International Working Group. Allergy 2014; 69: 602-16.

3. Longhurst HJ, Bork K. Hereditary angioedema: an update on causes, manifestations and treatment. Br J Hospital Med 2019; 80: 391-8.

4. Maurer M, Magerl M, Ansotegui I, et al. The international WAO/EAACl guideline for the management of hereditary angioedema - the 2017 revision and update. Allergy 2018; 73: 1575-96.

5. Farkas H, Martinez-Saguer I, Bork K, et al. International consensus of the diagnosis and management of pediatric patients with hereditary angioedema with C1inhibitor deficiency. Allergy 2017; 72: 300-13. 
6. Agostoni A, Aygoren-Pursun E, Binkley KE, et al. Hereditary and acquired angioedema: problems and progress: proceedings of the third C1 esterase inhibitor deficiency workshop and beyond. J Allergy Clin Immunol 2004; 114: S51-131.

7. Kaplan AP, Joseph K. Pathogenic mechanisms of bradykinin mediated diseases: dysregulation of an innate inflammatory pathway. Adv Immunol 2014; 121: 41-89.

8. Obtułowicz K. Bradykinin-mediated angioedema. Pol Arch Med Wewn 2016; 126: 76-85.

9. Cicardi M, Zuraw BL. Angioedema due to bradykinin dysregulation. J Allergy Clin Immunol Practice 2018; 6: 1132-41.

10. Ali MA, Borum ML. Hereditary angiooedema: what the gastroenterologist needs to know. Clin Exp Gastroenterol 2014; 7: 435-45.

11. Chen X, Yang YX, Liu YL, et al. Hereditary angioedema a rare cause of recurrent abdominal pain. Pak J Med Sci 2014; 30: 1147-9.

12. Bork K, Staubach P, Eckardt AJ, Hardt J. Symptoms, course, and complications of abdominal attacks in hereditary angioedema due to C1 inhibitor deficiency. Am J Gastroenterol 2006; 101: 619-27.

13. Locascio EJ, Mahler SA, Arnold TC. Intestinal angioedema misdiagnosed as recurrent episodes of gastroenteritis. Western J Emerg Med 2010; 11: 391-4.

14. Foix-L'Hélias L, Weiss L, Mollet-Boudjemline A, et al. Recurring acute abdominal pains in an adolescent as the presenting manifestations of hereditary angioneurotic oedema. Acta Paediatr 2005; 94: 1158-61.

15. Bork K, Aygören-Pürsün E, Bas M, et al. Guideline: hereditary angioedema due to C1 inhibitor deficiency. Allergo J Int 2019; 28: 16-29.

16. Rubinstein E, Stolz LE, Sheffer AL, et al. Abdominal attacks and treatment in hereditary angioedema with C1-inhibitor deficiency. BMC Gastroenterol 2014; 14: 71.

17. Riguzzi C, Losonczy L, Teismann N, et al. Gastrointestinal manifestations of hereditary angioedema diagnosed by ultrasound in the emergency department. West J Emerg Med 2014; 15: 816-8.

18. Patel N, Suarez LD, Kapur S, Bielory L. Hereditary angioedema and gastrointestinal complications: an extensive review of the literature. Case Rep Immunol 2015; 2015: 925861.

19. Bernstein JA, Cremonesi P, Hoffmann TK, Hollingsworth J. Angioedema in the emergency department: a practical guide to differential diagnosis and management. Int J Emerg Med 2017; 10: 15.

20. Brian T, Cheng BA, Jonathan I, et al. Burden of emergency department utilization and abdominal imaging for hereditary angioedema. J Allergy Clin Immunol Pract 2020; 8: 1443-6.e2.

21. Adhikari SP, Schneider JI. An unusual cause of abdominal pain and hypotension: angioedema of the bowel. J Emer Med 2009; 36: 23-5.

22. Czaller I, Molnár K, Csuka D, et al. Successful outcome using C1-inhibitor concentrate in acute pancreatitis caused by hereditary angioedema. Gastroenterol Nurs 2011; 34: 60-3.

23. Stobiecki M, Obtułowicz P, Porębski G, et al. Severe abdominal HAE attacks: an analysis of 7 cases. Arch Clin Med Case Rep 2019; 3: 527-33.

24. Gábos G, Moldovan D, Dobru D. Hereditary angioedema: a challenging diagnosis for the gastroenterologist. J Interdiscipl Med 2016; 1: 287-92.

25. Wasserman RL, Levy RJ, Bewtra AK, et al. Prospective study of $\mathrm{Cl}$ esterase inhibitor in the treatment of successive acute abdominal and facial hereditary angioedema attacks. Ann Allergy Asthma Immunol 2011; 106: 62-8.
26. Haneo MP, Kraschnewski J, Kelbel T, Craig T. Diagnosis and screening of patients with hereditary angioedema in primary care. Ther Clin Risk Manag 2016 12: 701-11.

27. Weinstock LB, Kothari T, Sharma RN, Rosenfeld SI. Recurrent abdominal pain as the sole manifestation of hereditary angioedema in multiple family members. Gastroenterology 1987; 93: 1116-8.

28. Kasamatsu Y, Yoshinoya K, Kasamatsu Y, et al. A case of hereditary angioedema involving recurrent abdominal attacks. Intern Med 2011; 50: 2911-4.

29. Nzeako U. Diagnosis and management of angioedema with abdominal involvement: a gastroenterology perspective. World J Gastroenterol 2010; 16: 4913-21.

30. Zanichelli A, Magerl M, Longhurst H, et al. Hereditary angioedema with $\mathrm{C} 1$ inhibitor deficiency: delay in diagnosis in Europe. Allergy Asthma Clin Immunol 2013; 9: 29.

31. Zanichelli A, Longhurst HJ, Maurer M, et al. Misdiagnosis trends in patients with hereditary angioedema from the real-world clinical setting Ann Allergy Asthma Immunol 2016; 117: 394-8.

32. Farkas H, Harmat G, Kaposi PN, et al. Ultrasonography in the diagnosis and monitoring of ascites in acute abdominal attacks of hereditary angioneutrotic oedema. Eur J Gastrol Hepatol 2001; 13: 1225-30.

33. Wakisaka M, Shuto $M$, Abe H, et al. Computed tomography of the gastrointestinal manifestation of hereditary angioedema. Radiat Med 2008; 26: 618-21.

34. Branco-Ferreira M, Pedro E, Barbos MA, Carols AG. Ascites in hereditary angioedema. Allergy 1998; 53: 543-5.

35. Obtulowicz P, Urbanik A, Obtulowicz K. Recurrent abdominal pain and ascites in patients suffering from congenital angioedema due to C1 inhibitor deficiency. Retrospective analysis. Przegl Lek 2013; 70: 299-302.

36. Jalaj S, Scolapio JS. Gastrointestinal manifestations, diagnosis, and management of hereditary angioedema. J Clin Gastroenterol 2013; 47: 817-23.

37. Goti F, Melcher GA, Spath P, Wuthrich B. Hereditary angioedema. A rare cause of acute abdominal pain with ascites. Dtsch Med Wochenschr 1998; 123: 1166-71.

38. Pedrosa M, Caballero T, Gómez-Traseira C, et al. Usefulness of abdominal ultrasonography in the follow-up of patients with hereditary C1-inhibitor deficiency. Ann Allergy Asthma Immunol 2009; 102: 483-6.

39. Ohsawa I, Nagamachi S, Suzuki H, et al. Leukocytosis and high hematocrit levels during abdominal attacks of hereditary angioedema. BMC Gastroenterol 2013; 13: 123.

40. Reshef A, Zanichelli A, Longhurst H, et al. Elevated D-dimers in attacks of hereditary angioedema are not associated with increased thrombotic risk. Allergy 2015; 70: 506-13.

41. Hofman ZLM, Relan A, Hack CE. C-reactive protein levels in hereditary angioedema. Clin Exp Immunol 2014; 177: 280-6. 